\title{
Pain symptomology, functional impact, and treatment of people with Neurofibromatosis type I
}

Frank D Buono'

Lauretta E Grau ${ }^{2}$

Matthew E Sprong ${ }^{3}$

Kenneth L Morford ${ }^{4}$

Kimberly J Johnson ${ }^{5}$

David H Gutmann ${ }^{6}$

'Department of Psychiatry, Yale School of Medicine, New Haven, CT, USA;

${ }^{2}$ Department of Epidemiology of Microbial Diseases, Yale School of Public Health, New Haven, CT, USA; ${ }^{3}$ Northern Illinois University, Dekalb, IL, USA;

${ }^{4}$ Department of Internal Medicine, Yale School of Medicine, New Haven, CT, USA; ${ }^{5}$ Institute of Public Health, Brown School, Washington University in St Louis, St Louis, MO, USA; ${ }^{6}$ Department of Neurology, Washington University School of Medicine, St. Louis, MO, USA
This article was published in the following Dove Press journal: Journal of Pain Research

Introduction: Neurofibromatosis type 1 (NF1) is a neurogenetic disorder affecting 1 in 3000 people worldwide, where individuals are prone to develop benign and malignant tumors. In addition, many people with NF1 complain of pain that limits their daily functioning. Due to the complexity of the disorder, there are few options for treating pain symptoms besides surgery and medications. Moreover, the spectrum of pain symptomatology and treatment, as well as the mechanisms underlying NF1-associated pain, has been understudied.

Methodology: To address this knowledge gap, we conducted a survey of 255 adults with NF1, leveraging the Washington University NF1 Patient Registry Initiative (NPRI) database. Demographic and pain data were collected using a Qualtrics survey.

Results: All participants had at least one surgical procedure, with 55\% reporting having at least one surgery within the last year and $17 \%$ being currently prescribed opioid medication. A positive relationship was shown $(p<0.001)$ between those prescribed prescription pain medication, and their pain severity and interference. Moreover, there was a significant relationship $(p=0.049)$ between the usage of complementary treatments and pain severity and interference.

Conclusion: The current study demonstrates that individuals with NF1 report a higher incidence of pain severity and interference than observed in NF1 previous studies, with pain symptoms not localized to any specific region of the body. The consideration for alternative treatments and careful monitoring of current treatments that are more conservative or have less potential adverse side effects may improve pain management and reduce the risk of developing medication dependence.

Keywords: Neurofibromatosis type 1, complementary treatment, adults, chronic pain

\section{Introduction}

Neurofibromatosis type 1 (NF1) is an autosomal dominant genetic condition, originally classified as a tumor predisposition syndrome based on the propensity of affected children and adults to develop benign and malignant tumors. ${ }^{1,2} \mathrm{NF} 1$ affects an estimated 1 in 3000 individuals worldwide, with half of all cases arising de novo with a known family history of the disorder. ${ }^{3}$ In addition to tumors, over $50 \%$ of individuals with NF1 report significant pain and discomfort. ${ }^{4,5}$ While this pain can be associated with tumors (neurofibromas), it is often not localized to a specific structural lesion, thus presenting treatment challenges for the individuals and their medical caregivers. ${ }^{6}$ Moreover, the increased pain in this population can negatively affect an individual's quality of life. ${ }^{7,8}$ Unfortunately, current therapeutic
Department of Psychiatry, Yale School of Medicine, 300 George Street, New

Haven, CT 065I0, USA

Tel +l 2038013499

Fax +I 2038013442

Email frank.buono@yale.edu 
options for treating pain are primarily limited to surgery or medication. ${ }^{9,10}$ In order to report on the effectiveness of therapeutic interventions aimed at improving NF1-associated pain, we utilized a large online database to survey adults with NF1 to (a) identify pain experience and symptoms, (b) evaluate its impact on function, and (c) determine the prevalence and complementary treatment modalities to reduce pain symptoms by surveying adults with NF1 using a large online database.

\section{Materials and methods}

\section{Participant recruitment and data collection procedures}

The Washington University Neurofibromatosis Type 1 Patient Registry Initiative (NPRI) was a web-based registry, launched in May 2011, collecting information on $>1000$ adults with NF1. For this study, participants were recruited between May 15, 2017, and June 1, 2017, through two direct e-mail invitations sent a week apart from the NPRI. Inclusion criteria consisted of (1) age 18 years or older and (2) currently enrolled in the NPRI registry. Potential participants received an e-mail message containing a recruiting script and a direct hyperlink to the survey. The survey site was hosted and maintained by Qualtrics (www.Qualtrics.com). Using the hyperlink, participants were requested to read the consent form and provide an electronic signature if they wanted to participate in the study. Participants had the option to refuse to answer any given item and were not obligated to complete the entire survey in one sitting. Participants had up to two weeks to complete the survey after consenting to participate. Following completion of the survey, participants were entered in a lottery to win one of four $\$ 50$ Amazon gift cards. The research protocol was approved by the Institutional Review Board at the Yale School of Medicine.

\section{Measures}

The survey consisted of demographic information, medical history, substance use questions, pain questions, and complementary alternative treatment questions. Demographic questions included age, gender, ethnicity, race, highest education, and marital status, while medical history items included number of surgeries in the last year, symptoms, and co-occurring disorders. Physician (D.H.G.) specializing in the treatment of NF1 and (K.M) specializes in addiction treatment provided input regarding the medical history items. The following assessments were added to the Qualtrics survey after the demographic section.
The NIDA ASSIST-Modified Quick Screen ${ }^{11}$ is a selfreport survey used to assess for frequency of use/abuse of substances over the past three months. There are seven multidimensional items, which are conditionally presented after a self-reported pre-screen is completed. The substances evaluated include cannabis, cocaine, prescription stimulants, methamphetamine, inhalants, sedatives, hallucinogens, street opioids, and prescription opioids. The NIDA ASSIST is considered highly reliable and valid.

The Brief Pain Inventory-Short Form (BPI-S) ${ }^{12}$ is a fifteen-question self-report Likert and open-ended survey to assess pain severity (worst, least, average, and right now) and interference in seven areas (mood, physical activity, work, social activity, relations with others, sleep, and enjoyment of life). The BPI-S scores range from 0 to 10 with higher scores indicating worse pain and lower scores indicating little to no pain. For pain interference and severity, all questions within each sub-category were aggregated and averaged. The reliability, construct validity, and sensitivity to changes over time have been validated in various studies assessing chronic pain. ${ }^{13}$

The complementary alternative treatment questions were adapted from Barry et $\mathrm{al}^{14}$ in collaboration with two chronic pain experts. The 27 open-ended questions were divided into two categories: evidence-based treatments and non-evidence-based complementary treatments as defined by the research through the National Center for Complementary and Integrative Health (NCCIH, 2017). Participants were asked about lifetime experience, as well as the past 30 days, for each complementary treatment (yoga, acupuncture, supplemental vitamins).

\section{Data analysis}

The estimated sample size of 115 was conducted a priori using $G^{*}$ Power, version 3.1.9.2 (Heinrich-Heine-Universitat Dusseldorf, Germany) where the effect size was 0.3 and the level of significance was set at $p=0.05$. All statistical analyses were computed using IBM SPS Statistics, version 24 (IBM Corp, Chicago, IL). The average completion rate across all outcome variables (ie, total pain score, number of surgeries) was $96 \%$ with rates ranging from $94 \%$ to $99 \%$. Descriptive statistics and confidence intervals were calculated and presented as sample characteristics. Associations between variables were examined using an unadjusted Pearson correlation coefficient and stepwise linear regression analysis, with an established significance set at $p=0.05$. The stepwise linear regression was implemented to control for homogeneity and correction for multiple comparisons. A stepwise regression 
analysis is a method in which several predictor variables are analyzed several times and each time the weakest predictor variable is removed from the model. ${ }^{15}$ Additionally, this model uses a combination of forward selection and backward elimination techniques. The forward selection model is a technique which analyzes several variables and only the strongest predictive variables are included in the model. The variables continue to be reanalyzed until no significant variables remain. In the backward elimination analysis, all variables are included in the model and the weakest predictive variables are removed, thus leading to variables remaining included in the model that are not necessary. After each step in the backward elimination model, in which a variable is added, all variables are reanalyzed to determine if the significance of the predictor variable has been reduced below a specific threshold.

\section{Results}

A total of 899 e-mails were sent using a large mail merge approach. Of those individuals who were e-mailed, 271 started the survey of whom 17 did not complete the survey within the allotted time leaving a total of 255 completed responses. Of the 255 participants, 61\% were female $(n=155)$ and $80 \%$ were Caucasian $(n=205)$ (Table 1). The mean age was $43.2(\mathrm{SD}=8.4)$ years, and on average, subjects were diagnosed with NF1 at the age of $11.9(\mathrm{SD}=12.1)$ years.

Most participants $(61 \%, \mathrm{n}=155)$ never smoked cigarettes, while $80 \%(n=205)$ currently do not smoke cigarettes. Of those who smoke, these individuals averaged $\sim 5$ cigarettes per day. Of the participants, 32\% $(n=81)$ used marijuana in their lifetime; $13 \%(n=32)$ used it in the last three months; $71 \%$ of subjects reported using it to relieve pain symptoms and to relax.

Subjects reported predominantly dermal neurofibromas $(73 \% ; n=186)$, and only $7 \%$ of subjects $(n=18)$ reported plexiform neurofibromas. Furthermore, 9\% $(n=22)$ of the current sample answered, "I do not know" when attempting to identify their specific tumors, indicating a potential lack of knowledge about their tumors. All participants had at least one surgical procedure to remove a tumor with a range of 1-34 surgical procedures; the average number of totals surgeries in an individual's lifetime being 4.9 $(\mathrm{SD}=5.1)$. In the last year, 55\% $(\mathrm{n}=140)$ reported having at least one surgery $(\mathrm{M}=1.73, \mathrm{SD}=0.87)$ of whom, $47 \%$ $(n=66)$ reported post-surgical complications within the last year, as defined by a physical issue (difficulty walking or lifting arm). Forty-three percent of subjects $(n=110)$ reported complications from all surgical procedures, with
$65 \%(n=71)$ of those describing permanent weakening of physical abilities following surgery.

A substantial proportion of adults with NF1 were prescribed prescription opioid medication $(55 \%, \mathrm{n}=139)$ in their lifetime to manage their pain symptoms (Table 2). In the last year, $17 \%(n=43)$ were currently prescribed opioid pain management treatment. When qualitatively asked to describe the effectiveness of their medication to treat their pain symptoms, $85 \%$ of those currently prescribed opioid medication indicated "little to know effectiveness". Furthermore, frequent somnolent effects were made ("made me too sleepy," "made me lazy"). Other prescribed medications used to relieve pain symptoms including GABA analogs (ie, pregabalin or gabapentin) and benzodiazepines (ie, Xanax, Valium) were equally ineffective. In this cohort, $27 \%(n=68)$ of adults were prescribed a GABA analog, $10 \%(n=25)$ were prescribed a benzodiazepine, and $51 \%(n=130)$ were actively using over-the-counter medications (OTC) to treat their pain symptoms. OTC medications were qualitatively rated above average in their effectiveness and side effects (ie, "at least I am not sleeping on the job with Tylenol," "I am still in pain but at least it is manageable").

Of the 255 participants, 220 (86\%) reported some level of pain (a minimum of 1) in the last three months, and nearly $95 \%(n=240)$ reported some level of pain during their lifetime. On average, participants reported living with pain for 11.2 years $(\mathrm{SD}=4.9)$, and given the complexity of NF1, many reported pain from tumors in several regions of their body (Table 1). The least affected area was the pelvic region (35\%); the most affected area was the arms (70\%). A stepwise linear regression analysis indicated that body regions (back, chest) nearly approached significance when evaluating the pain symptoms and number of surgeries $(p=0.054)$. Participants reported that multiple body areas were affected by tumors: $85 \%$ of individuals reported that more than two body areas were affected by tumors. There was a positive association between the number of affected areas and pain severity ratings $(p=<0.001)$. The average aggregate pain severity index was 4.7 , with an average aggregate pain interference of 4.9 , indicating moderate levels of daily pain symptoms. Of those who reported pain, 19\% $(n=42)$ of individuals reported moderately high pain severity and pain interference as defined by an aggregate score of 7 or higher. Stepwise linear regression analysis revealed a positive relationship between those taking prescription pain medication 
Table I Demographics and medical background of Neurofibromatosis type I subjects

\begin{tabular}{|c|c|}
\hline Demographics & $\mathbf{N}(\%)$ \\
\hline \multicolumn{2}{|l|}{ Gender } \\
\hline Male & $70(27)$ \\
\hline Female & $155(6 \mid)$ \\
\hline Transgender & $3(1)$ \\
\hline Age, Mean (SD) & $43.2(8.4)$ \\
\hline \multicolumn{2}{|l|}{ Ethnicity } \\
\hline Caucasian & $205(80)$ \\
\hline African American & $6(2)$ \\
\hline Hispanic & $7(2)$ \\
\hline Asian & $13(5)$ \\
\hline Native American & $14(5)$ \\
\hline Other & $10(4)$ \\
\hline \multicolumn{2}{|l|}{ Marital Status } \\
\hline Married & $105(4 I)$ \\
\hline Single & $96(38)$ \\
\hline Remarried & $I(I)$ \\
\hline Widowed & $6(2)$ \\
\hline Separated & $I(I)$ \\
\hline Divorced & $21(8)$ \\
\hline \multicolumn{2}{|l|}{ Education } \\
\hline High School or Less & $53(22)$ \\
\hline Some of College & $48(20)$ \\
\hline Associates/Technical Degree & $44(18)$ \\
\hline College Degree & $58(24)$ \\
\hline Advanced Degree & $40(16)$ \\
\hline Age of Diagnosis, Mean (SD) & $11.9(12.1)$ \\
\hline \multicolumn{2}{|l|}{ Medical Issues } \\
\hline Seizures & $16(6)$ \\
\hline Migraines & II4 (45) \\
\hline Scoliosis & $15(6)$ \\
\hline High Blood Pressure & $9(4)$ \\
\hline \multicolumn{2}{|l|}{ Body Parts Affected } \\
\hline Head & $162(64)$ \\
\hline Chest & $164(65)$ \\
\hline Face & $135(53)$ \\
\hline Back & $|3|(53)$ \\
\hline Arms & $177(70)$ \\
\hline Hands & $143(56)$ \\
\hline Torso & $156(6 \mathrm{I})$ \\
\hline Pelvis & $91(36)$ \\
\hline \multicolumn{2}{|l|}{ Type of Tumor } \\
\hline Dermal Neurofibromas & $176(70)$ \\
\hline Plexiform Neurofibromas & $17(7)$ \\
\hline Multiple Peripheral Nerve Sheath Tumors & $6(2)$ \\
\hline Neurofibromas & $14(5)$ \\
\hline Don't Know & $22(9)$ \\
\hline
\end{tabular}

Note: Due to missing data, all percentages may not add up to $100 \%$.
Table 2 Medications or substances to reduce pain symptoms

\begin{tabular}{|l|l|l|}
\hline \multirow{2}{*}{} & \multicolumn{2}{|l|}{ Treatments attempted, N (\%) } \\
\cline { 2 - 3 } & In the last 3 months & Lifetime \\
\hline Cigarette usage & $45(20)$ & $100(39)$ \\
Alcohol usage & $30(12)$ & $82(32)$ \\
Marijuana & $32(13)$ & $81(32)$ \\
Cocaine & $0(0)$ & $1(0)$ \\
Inhalants & $0(0)$ & $0(0)$ \\
Hallucinogens & $0(0)$ & $0(0)$ \\
Heroin & $0(0)$ & $0(0)$ \\
OTC pain relief & $130(5 \mathrm{I})$ & $18 \mathrm{I}(7 \mathrm{I})$ \\
GABA analogue & $68(27)$ & $150(59)$ \\
Benzodiazepines & $26(10)$ & $74(29)$ \\
Prescription opioids & $43(17)$ & $139(55)$ \\
\hline
\end{tabular}

and pain severity $(\mathrm{M}=5.1, \mathrm{SD}=1.5)$ or interference $(\mathrm{M}=6.2, \mathrm{SD}=2.1)$ relative to those who were not taking pain medications (severity $(\mathrm{M}=3.9, \mathrm{SD}=0.85)$, interference $[(\mathrm{M}=4.1, \mathrm{SD}=1.3), \mathrm{F}(3,108)=7.71, p<0.001])$.

In evaluating evidenced-based and non-evidencedbased complementary treatments (Table 3), the former was attempted less often than the latter within this population in the last three months. A Pearson correlation found a positive correlation between subjects currently using complementary treatments and pain severity $(p=0.021)$. There was a significant relationship between pain ratings' both severity and interference and the use of complementary treatments $[\mathrm{F}(3,97),=2.44, p=0.049]$.

\section{Discussion}

The primary aims of this study were to evaluate pain symptomology in a sample of adults with NF1 and to determine the frequency and perceived effectiveness of using integrated complementary treatment modalities to reduce pain symptoms. Leveraging the NPRI database, we analyzed pain symptomology and treatment in 255 adults with NF1. The findings in this report raise several important points.

First, our findings extend previous research by suggesting that a majority of individuals diagnosed with NF1 are dealing with tumors that produce pain and discomfort. ${ }^{4,5}$ Additionally, females in this sample reported higher pain interference (5.5) than males (4.3); however, males reported higher pain severity (5.1) than females (4.5). Furthermore, many of the individuals who were suffering with pain had been dealing with these symptoms for $>11$ years and reported higher pain thresholds, indicating pain 
Table 3 Complementary treatments usage

\begin{tabular}{|l|l|l|}
\hline \multirow{2}{*}{ Treatments } & \multicolumn{2}{l|}{$\begin{array}{l}\text { Treatments attempted, N } \\
\text { (\%) }\end{array}$} \\
\cline { 2 - 3 } & $\begin{array}{l}\text { In the last 3 } \\
\text { months }\end{array}$ & Lifetime \\
\hline Physical Exercise* & $97(38)$ & $154(60)$ \\
Chiropractor & $15(6)$ & $91(36)$ \\
Acupuncture* & $5(1)$ & $70(27)$ \\
Counseling/Therapy* & $9(4)$ & $56(22)$ \\
Yoga* & $22(9)$ & $57(22)$ \\
Physical therapy* & $26(10)$ & $95(37)$ \\
Meditation/Mindfulness* & $38(15)$ & $50(21)$ \\
Occupational therapy* & $5(2)$ & $58(22)$ \\
Supplemental vitamins & $49(19)$ & $107(42)$ \\
Self-help group & $26(10)$ & $105(4 I)$ \\
Hypnosis & $6(2)$ & $5 I(20)$ \\
Cold therapy & $4 I(16)$ & $75(29)$ \\
Prayer & $58(23)$ & $97(38)$ \\
Heat therapy & $54(24)$ & $78(3 \mathrm{I})$ \\
Transcutaneous electrical nerve & $25(10)$ & $51(20)$ \\
stimulation unit & & \\
\hline
\end{tabular}

Note: *Indicates the current treatment has been shown to evidenced based for chronic pain.

chronicity, along with multiple regions of their body being affected with NF1 tumors. Given the prevalence, duration and severity of pain symptoms within individuals of NF1 pain symptoms directly affect the quality of life of these individuals. Previous research has shown the impact of NF1 on quality of life ${ }^{8,16}$ and life expectancy; ${ }^{17}$ yet, further research is necessary to evaluate the relationship between pain severity and quality of life/expectancy. Moreover, a better understanding of the relationship between psychological disorders, ie, depression and anxiety, and pain symptoms within NF1, is needed.

Second, tumor-related pain currently is primarily managed by surgery, ${ }^{9,10}$ and can be problematic given NF1 tumors are by nerve tissues or other vital structures and tumor regrowth following surgery is common. ${ }^{1,18}$ All subjects reported in the current study had at least one surgical procedure to remove an NF1related tumor during their lifetime, and $27 \%$ had at least one surgical procedure within the last year. Also, $43 \%$ reported complications from all surgical procedures, with a majority of those suffering permanent weakening of physical abilities following the surgery, permanently affecting their activities of daily living and their quality of life. The prevalence with this population to utilize surgical management procedures that generally has more negative effects than positive indicates the severity of the issue, and lack of physically invasive procedures available for this population. These findings indicate that a majority of the current sample relied primarily on surgical procedures to manage pain symptoms and are consistent with those reported previously. ${ }^{9,10}$

Third, seventeen percent of subjects were actively taking opioids to manage pain symptoms, comparable to the national average of $20 \% .{ }^{19}$ Individuals who were taking opioids reported higher levels of pain severity and interference relative to those who were not prescribed these medications. Meldrum ${ }^{20}$ indicated chronic pain symptoms, specifically tumor-related, do not respond well to opioid medication; thus, providing an explanation for the negative qualitative comments on current opioid usage and initial justification for the difference in lifetime and current usage of opioid medication. Given the evidence that pain medications such as opioids seem to have little effect, with the risk for developing opioid-induced hyperalgesia, ${ }^{21}$ the promotion to access and gain knowledge of alternative treatments is needed.

Lastly, complementary treatments (yoga, massage therapy, physical therapy) have been shown to be effective with improvements in pain thresholds relative to non-evidence-based treatments. ${ }^{22}$ In other populations with a similar prevalence and co-occurring pain symptoms, such as cystic fibrosis and fibromyalgia, complementary treatments reduce the effects of chronic and acute pain symptoms. ${ }^{13,23,24}$ In the current study, a significant relationship was shown between pain ratings and usage of complementary treatments. In other words, with increased usage of complementary treatments, pain severity and interference scores decreased. The consideration for alternative treatments and careful monitoring of current treatments that are more conservative or have less potential adverse side effects may improve pain management and reduce the risk of developing medication dependence. While preliminary research on these treatments has been shown to be effective in adolescents with NF1, complementary and/ or alternative treatments have not been evaluated in adults with chronic pain symptoms. ${ }^{6,25}$ Future research will be required to evaluate the effectiveness of complementary treatments in adults with NF1 to suggest if that complementary treatment is more effective than pain medications. 
Given the complexity of the disease, understanding the potential mechanisms underlying pain symptoms for individuals in Neurofibromatosis would be inherently difficult. However, it should be noted that the current article attempted to bring attention to the chronicity of pain symptoms that can affect those with NF1. It should be noted, it is past the point of the manuscript to draw potential conclusions of these mechanisms without thoroughly understanding and evaluating how the clinical and psychologically implications can affect this population. Albeit, future studies should evaluate through a needs assessment of these mechanisms of pain.

Inherent to all patient-reported studies, this study has limitations. Despite the relatively large sample size for the given sample, it is a convenience sample and therefore of limited generalizability. Additional studies will be needed to confirm these findings. In addition, given the nature of the study, it is difficult to differentiate between pain due to presentation of tumors and that due to secondary symptoms caused by the tumors. Lastly, in the interest of reducing burden, we attempted to ensure the survey could have been completed in 30 mins. Past research has shown the survey length has been shown to increase respondent burden or fatigue. ${ }^{26,27}$

Future research is needed to further understand the rationale for individual's knowledge of their own disorder, while educating providers about this disorder. When evaluating the individual's own knowledge of NF1, $10 \%$ of the current sample did not know the type of tumor. Potential rationales for this could be recall biases, inadequate explanation by the provider, or unwillingness to deal with the disorder. Previous research has shown that individuals with uncommon or rare disorders are more prone to secrecy, ${ }^{28}$ less likely to divulge information to individuals, ${ }^{29}$ and less likely to personally understand the disorder due to inability to accept the disorder. Similarly, unless medical providers specialize in this disorder, understanding all facets of the disorder can be difficult especially when it comes to pain symptoms. ${ }^{18,30}$ Thus, it is critical to teach how to cope with these symptoms, while exposing both medical providers and individuals to the NF1.

\section{Conclusion}

NF1 is a lifelong medical condition in which chronic pain is a prominent feature. The current study demonstrates that individuals with NF1 report a higher incidence of pain severity and interference than observed in NF1 previous studies, with pain symptoms not localized to any specific region of the body. The lack of utilization of complementary treatments in this population needs to be further evaluated.

\section{Ethical approval and informed consent}

All procedures performed in studies involving human participants were in accordance with the ethical standards of the Yale School of Medicine Human Subjects Committee (IRB \#2000021068) and with the 1964 Helsinki declaration and its later amendments or comparable ethical standards. Informed consent was obtained from all individual participants included in the study.

\section{Acknowledgments}

We thank Dr Robert Kerns for his mentorship and recommendations and Ms Diana Haberkamp, of Neurofibromatosis Midwest, for allowing us to beta-test the survey within her organization. This study was not funded by any grants. Dr Matthew E Sprong is now affiliated with the Mental Health Services, Department of Veterans Affairs, Edward Hines, Jr. VA Hospital, Hines, IL, USA.

\section{Disclosure}

Dr David H. Gutmann reports a patent for Neurofibromin and mTOR issued to Washington University. Dr Gutmann also serves on the Editorial Board of Neuro-Oncology, Familial Cancer and GLIA, and receives license fee payments for the use of his GFAP-Cre mouse strain from the Tuberous Sclerosis Alliance. The authors report no other conflicts of interest in this work.

\section{References}

1. Anderson JL, Gutmann DH. Neurofibromatosis type 1. Handb Clin Neurol. 2015;132:75-86. doi:10.1016/B978-0-444-62702-5.00004-4

2. Hirbe AC, Gutmann DH. Neurofibromatosis type 1: a multidisciplinary approach to care. Lancet Neurol. 2014;13(8):834-843. doi:10.1016/ S1474-4422(14)70063-8

3. Gutmann DH, Ferner RE, Listernick RH, Korf BR, Wolters PL, Johnson KJ. Neurofibromatosis type 1. Nat Rev Dis Primers. 2017;3:17004. doi:10.1038/nrdp.2017.4

4. Brems H, Beert E, de Ravel T, Legius E. Mechanisms in the pathogenesis of malignant tumours in neurofibromatosis type 1. Lancet Oncol. 2009;10(5):508-515. doi:10.1016/S1470-2045(09)70033-6

5. Tucker T, Friedman JM, Friedrich RE, Wenzel R, Funsterer C, Mautner VF. Longitudinal study of neurofibromatosis 1 associated plexiform neurofibromas. $J$ Med Genet. 2009;46(2):81-85. doi:10.11 36/jmg.2008.061051 
6. Wolters PL, Burns KM, Martin S, et al. Pain interference in youth with neurofibromatosis type 1 and plexiform neurofibromas and relation to disease severity, social-emotional functioning, and quality of life. Am J Med Genet A. 2015;167A(9):2103-2113. doi:10.1002/ajmg.a.37123

7. Merker VL, Esparza S, Smith MJ, Stemmer-Rachamimov A, Plotkin SR. Clinical features of schwannomatosis: a retrospective analysis of 87 patients. Oncologist. 2012;17(10):1317-1322. doi:10.1634/theoncologist.2012-0162

8. Ferner RE, Thomas M, Mercer G, et al. Evaluation of quality of life in adults with neurofibromatosis 1 (NF1) using the Impact of NF1 on Quality Of Life (INF1-QOL) questionnaire. Health Qual Life Outcomes. 2017;15(34).

9. Creange A, Zeller J, Rostaing-Rigattieri S, et al. Neurological complications of neurofibromatosis type 1 in adulthood. Brain. 1999;122 (Pt 3):473-481. doi:10.1093/brain/122.3.473

10. Jeong YH, Choi EJ, Nahm FS. Concurrence of malignant peripheral nerve sheath tumor at the site of complex regional pain syndrome type 1 - a case report. Korean J Pain. 2013;26(2):160-163. doi:10.3344/kjp.2013.26.2.160

11. Smith PC, Schmidt SM, Allensworth-Davies D, Saitz R. A singlequestion screening test for drug use in primary care. Arch Intern Med. 2010;170(13):1155-1160. doi:10.1001/archinternmed.2010.140

12. Cleeland CS, Ryan K. Pain assessment: global use of the Brief Pain Inventory. Ann Acad Med Singapore. 1994;23(2):129-138.

13. Krebs EE, Bair MJ, Damush TM, Tu W, Wu J, Kroenke K. Comparative responsiveness of pain outcome measures among primary care patients with musculoskeletal pain. Med Care. 2010;48 (11):1007-1014. doi:10.1097/MLR.0b013e3181eaf835

14. Barry DT, Savant JD, Beitel M, et al. Use of conventional, complementary, and alternative treatments for pain among individuals seeking primary care treatment with buprenorphine-naloxone. J Addict Med. 2012;6(4):274-279. doi:10.1097/ADM.0b013e31826d1df3

15. Trochim W, Donnelly JP, Arora K. Research Methods: The Essential Knowledge Base. Boston, MA: Nelson Education; 2015.

16. Bicudo NP, de Menezes Neto BF, da Silva de Avo LR, Germano CM, Melo DG. Quality of life in adults with Neurofibromatosis 1 in Brazil. $J$ Genet Couns. 2016;25(5):1063-1074. doi:10.1007/s10897-016-9939-8

17. Rasmussen SA, Yang Q, Friedman JM. Mortality in neurofibromatosis 1: an analysis using U.S. death certificates. Am J Hum Genet. 2001;68(5):1110-1118. doi:10.1086/320121

18. Jett K, Friedman JM. Clinical and genetic aspects of neurofibromatosis 1 . Genet Med. 2010;12(1):1-11. doi:10.1097/GIM.0b013e3181bf15e3
19. Daubresse M, Chang HY, Yu Y, et al. Ambulatory diagnosis and treatment of nonmalignant pain in the United States, 2000-2010. Med Care. 2013;51 (10):870-878. doi:10.1097/MLR.0b013e3182a95d86

20. Meldrum ML. A capsule history of pain management. JAMA. 2003;290(18):2470-2475. doi:10.1001/jama.290.18.2470

21. Lee M, Silverman SM, Hansen H, Patel VB, Manchikanti L. A comprehensive review of opioid-induced hyperalgesia. Pain Physician. 2011;14(2):145-161.

22. Thomas DA, Maslin B, Legler A, Springer E, Asgerally A, Vadivelu N. Role of alternative therapies for chronic pain syndromes. Curr Pain Headache Rep. 2016;20(5):29. doi:10.1007/s11916-016-0562-z

23. Perry R, Leach V, Davies P, Penfold C, Ness A, Churchill R. An overview of systematic reviews of complementary and alternative therapies for fibromyalgia using both AMSTAR and ROBIS as quality assessment tools. Syst Rev. 2017;6(1):97. doi:10.1186/s13643017-0487-6

24. Zautra AJ, Davis MC, Reich JW, et al. Comparison of cognitive behavioral and mindfulness meditation interventions on adaptation to rheumatoid arthritis for patients with and without history of recurrent depression. J Consult Clin Psychol. 2008;76(3):408-421. doi:10.1037/0022-006X.76.3.408

25. Martin S, Wolters PL, Toledo-Tamula MA, et al. Acceptance and commitment therapy in youth with neurofibromatosis type 1 (NF1) and chronic pain and their parents: a pilot study of feasibility and preliminary efficacy. Am J Med Genet A. 2016;170(6):1462-1470. doi:10.1002/ajmg.a.37623

26. Sharp LM, Frankel J. Respondent burden: a test of some common assumptions. Public Opin Q. 1983;47:1. doi:10.1086/268765

27. Porter SR, Whitcomb ME, Weitzer WH. Multiple surveys of students and survey fatigue. New Directions Institutional Res. 2004;2004 (121):63-73. doi:10.1002/(ISSN)1536-075X

28. Zhu X, Smith RA, Parrott RL. Living with a rare health condition: the influence of a support community and public stigma on communication, stress, and available support. J Appl Commun Res. 2017;45 (2):179-198. doi:10.1080/00909882.2017.1288292

29. Pescosolido BA, Martin JK, McLeod JD, Rogers A. Handbook of the Sociology of Health, Illness, and Healing: A Blueprint for the 21st Century. New York (NY): Springer-Verlag; 2011.

30. Joachim G, Acorn S. Life with a rare chronic disease: the Scleroderma experience. J Adv Nurs. 2003;42(6):598-606.
Journal of Pain Research

\section{Publish your work in this journal}

The Journal of Pain Research is an international, peer reviewed, open access, online journal that welcomes laboratory and clinical findings in the fields of pain research and the prevention and management of pain. Original research, reviews, symposium reports, hypothesis formation and commentaries are all considered for publication. The manuscript

Submit your manuscript here: https://www.dovepress.com/journal-of-pain-research-journa management system is completely online and includes a very quick and fair peer-review system, which is all easy to use. Visit http:// www.dovepress.com/testimonials.php to read real quotes from published authors. 